\title{
Un modelo psicobiológico de la sobrealimentación inducida por estrés ${ }^{1}$
}

\section{A psychobiological framework to the stress-induced overeating}

Jaime R. Silva ${ }^{2}$

\section{Resumen}

Los dietantes crónicos muestran un patrón de alimentación paradójico: si bien restringen usualmente su consumo de alimentos, bajo ciertas situaciones se sobrealimentan. Se ha explicado que este patrón resulta de una estrategia cognitiva para controlar situaciones estresantes. La perspectiva de la neurociencia afectiva, desarrollada por Silva, sugiere que esta observación ocurre en individuos afectivamente vulnerables. Específicamente, las personas con una actividad frontal derecha incrementada son propensas a desarrollar aquellos patrones disfuncionales de control emocional. En este artículo se presenta un progreso teórico de este punto de vista científico. Bajo una aproximación psicobiológica, propongo que la sensibilidad al estrés es un aspecto esencial en la fisiología de los dietantes crónicos que puede ser entendida como una predisposición biológica a desarrollar alteraciones en la regulación emocional.

Palabras clave: dietantes crónicos, estrés, neurociencia afectiva.

\begin{abstract}
Restrained eaters show a paradoxical alimentary pattern: while usually restricts food consumption, under certain situation they overeat. This patter has been explained as a result of cognitive strategy to control stressful situation. The affective neuroscience perspective, developed by Silva, suggests that this observation occurs in affective vulnerable individuals. Specifically, subject with increased right frontal activity are prone to develop those patter of emotional control. In this paper, it is presented a theoretical progress from this scientific point of view. Under a psychobiological approach, I propose that stress sensitivity it is a key aspect of the restraint eaters' physiology that could be understand as a biological predisposition to develop difficulties in emotion regulation.

Key words: restrained eaters, stress, affective neuroscience.
\end{abstract}

1 Trabajo financiado por la Dirección de Investigación de la Universidad de La Frontera y FONDECYT 11090162.

2 Departamento de Salud Mental y Psiquiatría. Facultad de Medicina. Universidad de La Frontera. Temuco, Chile. jaimesilva@ufro.cl 


\section{Los dietantes crónicos o comedores emocionales}

Existe un grupo específico de individuos que por sus hábitos alimenticios han sido denominados comedores refrenados -restrained eaters- (Herman \& Polivy, 1980) o dietantes crónicos (Silva, 2003, 2005a). Estas personas se caracterizan por experimentar un temor exacerbado a ganar peso, restringiendo su alimentación por medio de dietas. Paradójicamente, bajo ciertas condiciones estos individuos aumentan sus niveles de ingesta, sobrealimentándose. Se ha observado desinhibición alimenticia en dietantes crónicos expuestos a eventos estresantes (Cools, Schotte \& McNally, 1992; Heatherton, Herman \& Polivy, 1991; Herman \& Polivy, 1975; Polivy, Herman \& McFarlane, 1994; Schotte, Cools \& McNally, 1990), tareas cognitivas demandantes de atención (Ward \& Mann, 2000) y a diversos estímulos conectados a distintos tipos de estímulos alimentarios (Herman \& Mack, 1975; Jansen, Merckelbach, Oosterlaan, Tuiten \& Van den Hout, 1988; Spencer \& Fremouw, 1979; Wardle, Steptoe, Oliver \& Lipsey, 2000). Si bien la restricción crónica de la alimentación no representa un trastorno psicopatológico (al menos reconocido como tal en los manuales de diagnóstico tradicionales), es un factor de riesgo importante para el desarrollo de los desórdenes de la alimentación (Jacobi, Hayward, de Zwaan, Kraemer \& Agras, 2004; Keel, Baxter, Heatherton \& Joiner, 2007), y también de la obesidad (Jebb, 1997; Rennie, Johnson \& Jebb, 2005). De hecho, cabe la posibilidad de que la restricción alimenticia y sus potenciales consecuencias (especialmente en condiciones de estrés crónico), conformen una sucesión de estadios hacia el desarrollo de la obesidad (Silva, 2007b). Al parecer, los fenómenos psiconeuroendocrinológicos que se originan a partir del estrés crónico, y que se encuentran acentuados especialmente en personas crónicamente restringidas en su alimentación, favorecen el desarrollo progresivo de aumento de peso (obesidad central) y la cascada de eventos derivados.

Se han propuesto varios modelos explicativos de la conducta alimenticia del dietante crónico (DC), especialmente de la combinación particular de restricción/sobrealimentación. Polivy y Herman (1999) sostienen que los DC atribuyen, erróneamente, la ansiedad relacionada con la amenaza de la autoestima y es reinterpretada como angustia por 
haber roto la dieta. La ansiedad estaría aún presente, pero reinterpretada de una manera preferible; de una situación vivida como sin control a una situación potencialmente controlable ("mañana empiezo la dieta"). Por esta razón, estos autores afirman que el quiebre de restricción alimenticia es una forma de "enmascarar" la ansiedad y la amenaza al self. En conjunto, los estudios en esta línea de pensamiento muestran que los dietantes crónicos requieren modular sus estados afectivos negativos asociados a la amenaza de la auto-imagen, enmascarando el origen de la ansiedad, de la atribución de esos estados negativos al rompimiento de la dieta. Sin embargo, este mecanismo no tendría como resultado la eliminación de la experiencia emocional negativa sino que probablemente modifica la sensación de control sobre el evento adverso, de ser una situación incontrolable (el fracaso) a una controlable (dieta) (Baumeister, 1991).

\section{Estilo afectivo y restricción alimenticia crónica: un modelo de la neurociencia afectiva}

En el contexto antes descrito, el equipo de investigación liderado por Silva (Silva, 2002, 2003, 2005a, 2005b, 2005c, 2005d, 2007b, 2008a, 2008b, en prensa; Silva, Livacic-Rojas \& Slachevsky, 2006; Silva \& Pizzagalli, 2006; Silva, Pizzagalli, Larson, Jackson \& Davidson, 2002; Silva \& Slachevsky, 2005), ha utilizado paradigmas teóricos/empíricos de la neurociencia afectiva, que en el marco de estudios experimentales, correlacionales y de campo, ha permitido la elaboración de un modelo de la influencia del estilo afectivo en la conformación del patrón alimenticio alterado del DC.

En esta sección se describirá en detalle la aproximación mencionada. El aspecto central de la propuesta aquí formulada se basa en dos estudios relacionados que exploran la relación entre la asimetría de los sistemas motivacionales y la restricción alimentaria crónica. El primero de ellos, realizado en el Laboratory for Affective Neuroscience (Silva, et al., 2002), mostró que existe una relación entre la asimetría frontal funcional y los niveles de restricción alimentaria de un individuo. En este contexto, se demostró que los DC pueden caracterizarse como sujetos con una asimetría funcional derecha de la corteza prefrontal (CPF). Considerando el modelo 
de aproximación-evitación (Davidson, 1998, 2002, 2004; Davidson, Ekman, Saron, Senulis \& Friesen, 1990; Silva, 2003, 2005c; Tomarken $\&$ Keener, 1998) lo anterior indicaría que las personas que realizan dieta crónicamente poseen un sistema motivacional de inhibición más activo, es decir, una tendencia exacerbada a elaborar representaciones mentales que anticipan eventos amenazantes (componente prospectivo), una tendencia a mantener en la memoria de trabajo estos contenidos (componente retrospectivo) y, por ende, una inhibición de representaciones asociadas al reforzamiento. Esto sugiere que los comedores emocionales tienen un estilo afectivo vulnerable, el que debería implicar una sensibilidad al estrés, un bajo umbral de activación de la ansiedad y una dificultad en regular las emociones negativas (Silva, 2003).

En este contexto, el mecanismo de enmascaramiento descrito por Polivy y Herman (Polivy \& Herman, 1999; Silva, 2007b), sería un modo aprendido de regular las disposiciones emocionales exacerbadas. En otras palabras, en el DC se daría una combinación entre un estilo afectivo vulnerable en conjunto con un mecanismo de regulación específico (alternancia de la restricción/sobrealimentación).

En un segundo reporte, Silva y colaboradores (2006) realizaron una inducción experimental de sobrealimentación y observaron el comportamiento de los sujetos considerando las asimetrías de los sistemas motivacionales. Tal como predice el modelo de aproximación-evitación, los comedores emocionales con un sistema de evitación sensibilizado tienden a fracasar en su restricción alimentaria. Adicionalmente, el estudio sugiere que los DC con un sistema de aproximación más sensible tienen un patrón alimentario opuesto, es decir, se restringen más que los sujetos normales. De este modo, las diferencias individuales en los DC estarían en un nivel asociadas a la influencia de los sistemas motivacionales en la restricciónsobrealimentación. Por ello, tal como se ha sostenido (Silva, 2005a), esta conceptualización permite especular la presencia de sendas divergentes de desarrollo de alteraciones alimentarias en los DC dependientes de la sensibilidad del sistema motivacional de aproximación o evitación. Así, el DC con un sistema de evitación más activo sería aquel que, bajo condiciones crónicas de estrés, presentaría la secuencia de estadios para 
el desarrollo de obesidad (Silva, 2007b), debido a que su estilo afectivo favorecedor de experiencias de ansiedad requeriría permanentemente una modulación de dichas oscilaciones emocionales a través del mecanismo de enmascaramiento y, por ende, de la sobrealimentación. Por otro lado, los DC con un sistema de aproximación más activo modularían el estrés crónico manteniendo sus metas prospectivas y retrospectivas asociadas al refuerzo (hacer dieta), aumentando la probabilidad de sufrir trastornos alimentarios en la línea de la Anorexia Nervosa.

Las observaciones antes descritas deben complementarse con dos estudios del programa de investigación en revisión (Silva, 2007a; Silva, Jiménez, Sapunar \& Larenas, 2007; Silva \& Pizzagalli, 2006). El reporte de Silva y Pizzagalli (2006), incluyó un paradigma de inducción de ansiedad en el cual los participantes debían observar estímulos de tres clases: neutros, aversivos y alimenticios. En tales condiciones emocionales, los DC mostraron un procesamiento cortical de los estímulos alimenticios anormal, lo cual es particularmente afín a la hipótesis de enmascaramiento. Efectivamente, a diferencia de los controles, en individuos DC los estímulos alimenticios reclutan estructuras corticales de la línea media, regiones que están implicadas en el procesamiento auto-referencial de estímulos y la auto-imagen (Northoff \& Bermpohl, 2004; Northoff, et al., 2006; Torres, Silva, J.R. \& Silva, F.L., 2007). Adicionalmente, este procesamiento se acompañó con una sensibilización de las redes occipitales relacionadas con la atención motivada. Es por ello que Silva y Pizzagalli (2006) sugieren que "el proceso auto-referencial activado en respuesta a la comida sería un aspecto esencial del procesamiento de información de los DC en condiciones de ansiedad".

Finalmente, en un estudio preliminar de potenciales cerebrales (Silva, Jiménez, Sapunar y Larenas, 2007; Silva, 2007b), nuestro equipo de investigación abordó un aspecto esencial de la respuesta emocional asociado a la psicopatología; la diferenciación emocional (Silva, 2003, 2004, 2005d). Considerando la creciente evidencia que sostiene que la diferenciación emocional es un aspecto esencial en la regulación afectiva (Barrett, Mesquita, Ochsner \& Gross, 2007) y el desarrollo de alteraciones de la alimentación como el atracón alimenticio (Van Strien 
\& Ouwens, 2007), se intentó abordar esta noción desde una perspectiva psicofisiológica en sujetos DC. Para ello se utilizó un paradigma experimental que incluye la presentación de rostros emocionalmente expresivos, el cual induce en sujetos normales una respuesta cortical occipital/temporal diferenciada para expresiones neutras, positivas y negativas (Krolak-Salmon, Fischer, Vighetto \& Mauguiere, 2001). Esta respuesta cerebral es claramente observable alrededor de los $250 \mathrm{~ms}$. para la categoría neutro versus emoción, y alrededor de los $550 \mathrm{~ms}$. para la categoría neutro, emoción positiva y emoción negativa. En este contexto, en concordancia con las hipótesis del estudio, los sujetos DC presentaron un patrón cortical indiferenciado frente a las distintas clases de expresiones emocionales en todas las ventanas temporales de interés. Esta es la primera evidencia psicofisiológica que muestra que los DC presentan dificultades en su habilidad para diferenciar emociones, lo cual en conjunto con las características mencionadas anteriormente, conformarían un perfil de estilo afectivo que favorece el desarrollo de mecanismos disfuncionales de modulación afectiva (sobrealimentación/restricción).

En síntesis, el modelo neurocientífico presentado propone que los DC son individuos cuyo estilo afectivo vulnerable explica parte de sus propensiones características, en especial a experimentar emociones asociadas al sistema motivacional sensibilizado y su necesidad permanente de regular los afectos negativos. En este contexto un grupo importante de DC posee un sistema de motivación de evitación preponderante, lo que conlleva una tendencia exacerbada a anticipar los eventos futuros como amenazantes y experimentar ansiedad en respuesta a ello. Asimismo, el estilo afectivo de los DC incluiría un procesamiento alterado de los estímulos alimentarios y deficiencias en la diferenciación emocional (Silva, 2008b).

En este marco, se entiende el enmascaramiento como una estrategia de regulación emocional precaria tendiente a afrontar su vulnerabilidad emocional. El estilo afectivo representaría una diátesis genérica para el desarrollo de diferentes trastornos psicopatológicos, incluyendo los trastornos de la alimentación y la obesidad. Dependiendo de las experiencias del sujeto, especialmente las condiciones de estrés (agudo o crónico), dicha 
vulnerabilidad se expresaría en un afecto negativo y una dificultad en modular su intensidad. El nivel de diferenciación emocional determinaría las condiciones sobre las cuales elaborar estrategias de regulación afectiva, donde a menor nivel de diferenciación mayor probabilidad de elaboración de estrategias disfuncionales (entre ellas la alternancia sobrealimentaciónrestricción).

\section{¿Es la sensibilidad al reforzamiento un mecanismo inductor de la sobrealimentación en los DC?: hacia la formulación de un programa de investigación alternativo}

La conducta alimentaria deriva de la conjunción compleja de procesos cognitivos, emocionales, metabólicos y neuroendocrinos, por nombrar algunos. Está más allá de las posibilidades de este artículo revisar en detalle la evidencia en relación a ello. Sin embargo, algunos elementos del sustrato biológico de la alimentación han recibido mayor atención de parte de los investigadores de la alteración de la conducta alimentaria. En efecto, una de las hipótesis psicobiológicas en el campo de la obesidad y los desórdenes alimentarios que más fuerza ha cobrado recientemente es la sensibilidad al reforzamiento como mecanismo inductor de la sobrealimentación (Beaver, et al., 2006; Berthoud \& Morrison, 2008; Davis, Curtis, Tweed \& Patte, 2007; Davis \& Fox, 2008; Davis, et al., 2008; Davis, Patte, et al., 2007; Davis, Strachan \& Berkson, 2004; Finlayson, King \& Blundell, 2007; Gibson, 2006; Guerrieri, Nederkoorn \& Jansen, 2008; Nasser, 2001; Nederkoorn, Braet, Van Eijs, Tanghe \& Jansen, 2006; Wang, Volkow, Thanos \& Fowler, 2004).

Se ha asumido que una reactividad anormal de los sistemas cerebrales asociados al refuerzo explicaría las causas y/o consecuencias de las conductas alimentarias alteradas, como el atracón alimentario (binge eating). La vieja escuela que considera la obesidad como "externamente" instigada (Schachter, 1968), encuentra un renovado sustento en estas ideas. Aun cuando estos sistemas puedan descomponerse para su estudio, lo cierto es que son una red distribuida en todo el cerebro, y por ende, tal como expresan Berthoud y Morrison (2008), "el substrato neurológico responsable de la experiencia de placer asociado a la alimentación es complejo y distribuido 
a través del eje neural, y no puede ser apropiadamente eliminado mediante lesiones de tejido de los componentes de la red” (p. 75). Así, tanto en modelo animal como en humanos, la investigación en este ámbito no ha sido del todo sencilla. En este contexto, se ha dado especial importancia al valor hedónico (el llamado "liking") y a la saliencia del estímulo (el "wanting") (Berridge \& Kringelbach, 2008). Ambos aspectos serían clave en la inducción de la conducta de alimentación, y su exacerbación se correlacionaría respectivamente con (a) una elevada experiencia de placer asociada al alimento y (b) una sensibilidad aumentada a responder frente a estímulos alimentarios comiendo.

El sistema cerebral relacionado con este tipo de conductas muestra una segregación en correspondencia con aquello: por un lado, el valor hedónico parece depender de un sistema cerebral distribuido, incluyendo el cerebro anterior límbico y el tallo cerebral, cuyo "centro principal" deriva de la activación $\mu$-opioide en la concha del nucleus accumbens (Pecina, 2008; Pecina \& Berridge, 2000). Por otro lado, la saliencia del estímulo depende fundamentalmente de las proyecciones dopaminérgicas del área tegmentada ventral hacia el nucleus accumbens y del hipotálamo lateral (Pecina, 2008). En la corteza cerebral los aspectos cognitivoemocionales complejos se han relacionado con la CPF, especialmente la CPF orbitofrontal (Grabenhorst, Rolls \& Bilderbeck, 2007; Grabenhorst, Rolls, Margot, Da Silva \& Velazco, 2007; Kringelbach, O’Doherty, Rolls \& Andrews, 2003; Rolls, 1996, 2004), tanto en estudios con personas normales como en personas con daño cerebral.

Asimismo, Pizzagalli y colaboradores (2005), mostraron en un estudio particularmente relevante para este análisis, que la sensibilidad al reforzamiento está mediada por la activación de la corteza prefrontal, siendo la actividad tónica de la CPF izquierda la responsable del 53\% de la varianza del sesgo en favor del refuerzo. Si bien es indudable que una de las vertientes de la obesidad y los desórdenes alimentarios deriva efectivamente en la alteración del sistema cerebral del reforzamiento, ello no armoniza con la evidencia recolectada por los estudios cognitivos ni con el modelo de la neurociencia afectiva para los DC. Efectivamente, tal como se describió en las secciones anteriores, los DC tienen un comportamiento 
alimentario anormal derivado de un mecanismo de regulación de estados emocionales negativos, el cual no depende del valor hedónico del alimento ni de su saliencia: por un lado, como se mencionó, la sobrealimentación del DC no se relaciona a la palatabilidad del alimento (Polivy et al., 1994) y, por otro, se ha insistido que frecuentemente la conducta de atracón alimentario no está conectada a la sensación de hambre (Greeno, Wing \& Shiffman, 2000; Waters, Hill \& Waller, 2001). Es más, en el estudio de Silva \& Pizzagalli (2006), la observación de una hipoactivación de la corteza prefrontal subgenual, estructura asociada al sistema motivacional apetitivo (Small, Zatorre, Dagher, Evans \& Jones-Gotman, 2001), parece indicar que en una situación de estrés el procesamiento inicial de los estímulos alimentarios no se conecta a una experiencia placentera en los DC. Más aún, según el modelo de la neurociencia afectiva, la asimetría frontal derecha (que favorece la sensibilidad al estrés) se asocia a los DC donde predomina la sobrealimentación, mientras que la asimetría izquierda (que favorece la sensibilidad al reforzamiento) se relacionaría con los DC más restrictivos (Silva et al., 2006). En acuerdo a este último punto, el estudio de Brunstrom y colaboradores (2004) mostró que los dietantes que se sobrealimentan no muestran un patrón exacerbado de salivación frente al alimento (como podría predecir el modelo de sensibilidad al reforzamiento), mientras que aquellos restrictivos sí.

En su conjunto, estas observaciones cuestionan la generabilidad de la hipótesis de la sensibilidad al reforzamiento como factor inductor de la conducta alimentaria alterada en un subgrupo de personas con tendencia a desarrollar desórdenes alimentarios u obesidad (DC o comedores emocionales). Considerando que tanto la obesidad como los desórdenes alimentarios probablemente son el punto de convergencia de diversas sendas etiológicas, entonces se requiere explicar científicamente cada una de ellas. Los DC, como parte de este grupo, parecen no mostrar la sensibilidad al reforzamiento en su perfil psicofisiológico y, por ende, es necesario otro paradigma para comprender su alteración.

En la siguiente sección se elaborará una perspectiva alternativa, la cual considera para su formulación la evidencia cognitiva, emocional, 
neuroendocrina y el modelo de Silva (Silva, 2008a, 2008b), estableciendo los fundamentos que sustentan una perspectiva novedosa en el campo de estudio presentado.

\section{La sensibilidad al estrés y el factor de liberación de corticotrofina (CRF): una perspectiva alternativa de la conducta alimentaria alterada en los DC}

Uno de los componentes esenciales de la fisiología de la respuesta al estrés es el factor de liberación de corticotrofina (CRF) y sus receptores asociados (CRF1 y CRF2), los cuales regulan y modulan el eje hipotálamopituitario-adrenal y la respuesta del sistema nervioso central frente a éste (Bale \& Vale, 2004; Heinrichs, Menzaghi, Merlo Pich, Britton \& Koob, 1995; Smagin \& Dunn, 2000). En el eje HPA, el CFR es secretado por el núcleo paraventricular del hipotálamo, induciendo la liberación de ACTH en los corticotropos de la glándula pituitaria anterior. Luego, el ACTH estimulará la síntesis y liberación de glucocorticoides en la glándula adrenal. Éstos producirán un feedback negativo, inhibiendo la producción y liberación de CFR en el hipotálamo. A nivel central, el estrés también favorece la secreción de CRF, el cual activa el sistema nervioso autónomo y facilita la liberación de norepinefrina y epinefrina en la circulación. Esta cascada de procesos media la respuesta conductual, cognitiva, autonómica, neuroendocrina e inmunológica frente al estrés (Heinrichs, et al., 1995).

Sin embargo, se ha descrito que algunas personas muestran una hiper-reactividad en los sistemas de estrés, lo cual se relacionaría con trastornos ansiosos, y con desórdenes afectivos en general (Boyce \& Ellis, 2005; Heinrichs, et al., 1995). Incluso, se ha planteado que la sensibilidad al estrés es un aspecto común a este tipo de patologías (Bale, 2006; Bale \& Vale, 2004). Consecuentemente, en varios estudios las disfunciones de la afectividad se relacionan con una hiperactividad de los sistemas centrales de CRF (incluyendo hipersecreción de CRF) y/o un funcionamiento alterado del eje HPA (Helmreich, Parfitt, Lu, Akil \& Watson, 2005; Hughes, Watkins, Blumenthal, Kuhn \& Sherwood, 2004; Krysiak, Obuchowicz \& Herman, 2000; Olff, Guzelcan, de Vries, Assies \& Gersons, 2006; Pardon, Ma \& Morilak, 2003; Rosmond, 2003; Shekhar, Truitt, Rainnie 
\& Sajdyk, 2005; Starkman, Cameron, Nesse \& Zelnik, 1990; Takahashi, 2002; Todorovic, Jahn, Tezval, Hippel \& Spiess, 2005; Wyatt, Portnoy, Kupfer, Snyder \& Engelman, 1971).

Interesantemente, tanto en el atracón alimentario (Gluck, Geliebter, Hung \& Yahav, 2004), la bulimia (Koo-Loeb, Costello, Light \& Girdler, 2000), la anorexia (Brown, Camilleri, Go, et al., 1987) y la obesidad (Marin, Darin, Amemiya, Andersson, Jern, \& Bjorntorp, 1992) se han reportado niveles elevados de reactividad fisiológica frente a estresores. Asimismo, algunos estudios han enfatizado la relación entre cortisol, estrés y sobrealimentación. Por ejemplo, Epel, Lapidus, McEwen y Brownell (2001), encontraron en un grupo de personas normales que aquellas con niveles elevados de cortisol en respuesta a un estresor se sobrealimentaban. En modelo animal, el grupo de Dallmann (Dallman, 2007; Dallman, Akana, Strack, Hanson \& Sebastian, 1995; Dallman, et al., 2004; Dallman, et al., 2003; Dallman, et al., 2006) ha desarrollado un elegante paradigma donde se enfatiza que la elevación crónica de glucocorticoides derivada de la exposición crónica al estrés resulta en la estimulación de conductas mediadas por las vías dopaminérgicas mesolímbicas del reforzamiento, incrementando la alimentación (entre otros efectos). Similarmente, en un estudio reciente (Pecina, Schulkin \& Berridge, 2006), de alta relevancia para la perspectiva aquí asumida, se realizaron microinyecciones de CRF en la concha del accumbens medial de ratas de laboratorio. Los resultados sugieren que el estrés paradójicamente puede inducir la sensibilización del sistema de refuerzo. En palabras de Pecina y colaboradores, "nuestros resultados sugieren que aunque el estrés y el CRF hipotalámico tienen un efecto motivacional predominantemente negativo, la activación de los sistemas de CRF en la concha del accumbens medial pueden efectivamente favorecer la conducta apetitiva a través de un efecto motivacional positivo. Esto ofrece una explicación novedosa del por qué el estrés puede, en ocasiones, exacerbar el atracón alimentario, las recaídas a la adicción a drogas y otras búsquedas excesivas de reforzamiento" (Pecina et al., 2006, p.12). Por lo tanto, la sensibilidad al estrés puede resultar en modificaciones de la saliencia de los estímulos positivos como el alimento. Aún así, desde esta perspectiva, el aspecto esencial en la inducción de la conducta alimentaria 
es la respuesta neurofisiológica al estrés, ya que ésta sería la instigadora de la cascada de procesos que resulta en la sobrealimentación.

El modelo de la neurociencia afectiva (Silva, 2007a, 2008a, 2008b) sostiene que los DC son individuos con un estilo afectivo vulnerable, que incluye un sistema motivacional de evitación más activo. Este sistema favorece la experiencia emocional de ansiedad y se le ha relacionado con tendencias al desarrollo de desórdenes del ánimo (Blackhart, Minnix \& Kline, 2006; Buss, et al., 2003; Coan \& Allen, 2003; Davidson, 2002, 2004; Davidson, Lewis, et al., 2002; Davidson, Marshall, Tomarken \& Henriques, 2000; Davidson, Pizzagalli, Nitschke \& Putnam, 2002; Sutton $\&$ Davidson, 2000). Se ha sostenido que los individuos con este sistema hiperactivo tienen asociado a ello un bajo umbral de activación de los sistemas fisiológicos responsables de la respuesta de estrés (Amodio, Master, Yee \& Taylor, 2008; Buss, et al., 2003; Davidson, 2004; Kalin, Larson, Shelton \& Davidson, 1998; Scholten, Van Honk, Aleman \& Kahn, 2006), de tal forma que se espera que un estresor resulte en respuestas fisiológicas exacerbadas en estos sujetos.

Aun cuando las conductas de desinhibición alimentaria en los DC se observan justamente en respuesta a situaciones inductoras de estrés, no existe hasta ahora ninguna evidencia experimental que indique que; (a) las respuestas de ansiedad de los DC en algún parámetro (conductual, autoreporte, psicofisiológicos) son más intensa; y (b) su sistema fisiológico presente respuestas exacerbadas en contextos inductores de ansiedad. Hasta donde conocemos, no existe ningún estudio en DC que indague la sensibilidad al estrés, en condiciones de inducción de ansiedad y desde una perspectiva neuro-endocrino-psicofisiológica -existe un reporte (Tuschen-Caffier \& Vogele, 1999) donde se evaluaron sólo medidas psicofisiológicas pero se utilizó un inductor de ansiedad imaginario, lo que cuestiona la validez del experimento-. Asimismo, nadie hasta ahora ha puesto a prueba la hipótesis de la sensibilidad al reforzamiento en DC, bajo paradigmas experimentales que confiablemente recluten ese sistema motivacional.

El grupo de investigación que he liderado ha comenzado a explorar esta noción formulando dos proyectos inéditos. En el primero de ellos, 
titulado "Asociación de polimorfismos del gen CRF-BP en individuos dietantes crónicos y su relación con un patrón de conducta" (DIUFRO, DI09-0043), a través de una perspectiva genético-conductual, se pretende probar que los DC muestran polimorfismos del gen de CRF-BP, lo cual tendría relación con la desregulación del eje HPA y la tolerancia a los agentes estresores. Sin embargo, se requiere evidencia en otros niveles de análisis, tal como se expuso en esta sección, especialmente respecto de la respuesta fisiológica al estrés y la modulación psicofisiológica relacionada con el reforzamiento. Así, en el segundo proyecto en curso (FONDECYT 11090162) el desafío es recolectar evidencia para responder la siguiente pregunta de investigación: ¿Es la sensibilidad al reforzamiento o sensibilidad al estrés lo que caracteriza el estilo afectivo de los dietantes crónicos? Para lograr este objetivo se debe: (1) Probar si existen diferencias en las respuestas psicofisiológica y conductual de los DC versus los NDC en un paradigma que recluta al sistema motivacional de reforzamiento, evaluando además si el estrés agudo es capaz de modular dichas respuestas a favor de uno u otro sistema motivacional; (2) Probar en los DC si algunos parámetros neuroendocrinos, psicofisiológicos y/o conductuales, bajo condiciones de inducción experimental de ansiedad, reflejan una sensibilidad aumentada del sistema neuro-endocrino-psicofisiológico del estrés.

\section{Referencias bibliográficas}

Abell, T. L., Malagelada, J. R., Lucas, A. R., Brown, M. L., Camilleri, M., Go, V. L., et al. (1987). Gastric electromechanical and neurohormonal function in anorexia nervosa. Gastroenterology, 93(5), 958-965.

Amodio, D. M., Master, S. L., Yee, C. M. \& Taylor, S. E. (2008). Neurocognitive components of the behavioral inhibition and activation systems: Implications for theories of self-regulation. Psychophysiology, 45(1), 11-19.

Bale, T. L. (2006). Stress sensitivity and the development of affective disorders. Horm Behav, 50(4), 529-533.

Bale, T. L. \& VAlE, W. W. (2004). CRF and CRF receptors: role in stress responsivity and other behaviors. Annu Rev Pharmacol Toxicol, 44, 525-557. 
Barrett, L. F., Mesquita, B., Ochsner, K. N. \& Gross, J. J. (2007). The experience of emotion. Annu Rev Psychol, 58, 373-403.

Baumeister, R. F. (1991). Escaping the Self: Alcoholism, Spirituality Masochism, and Others Flights from the Burden of Selfhood. New York: Basic Books.

Beaver, J. D., Lawrence, A. D., van Ditzhuijzen, J., Davis, M. H., Woods, A. \& Calder, A. J. (2006). Individual differences in reward drive predict neural responses to images of food. J Neurosci, 26(19), 5160-5166.

Berridge, K. C. \& Kringelbach, M. L. (2008). Affective neuroscience of pleasure: reward in humans and animals. Psychopharmacology, 199(3), 457-480.

Berthoud, H. R. \& Morrison, C. (2008). The brain, appetite, and obesity. Annu Rev Psychol, 59, 55-92.

Blackhart, G. C., Minnix, J. A. \& Kline, J. P. (2006). Can EEG asymmetry patterns predict future development of anxiety and depression? A preliminary study. Biol Psychol, 72(1), 46-50.

Boyce, W. T. \& Ellis, B. J. (2005). Biological sensitivity to context: I. An evolutionary-developmental theory of the origins and functions of stress reactivity. Dev Psychopathol, 17(2), 271-301.

Brunstrom, J. M., Yates, H. M. \& Witcomb, G. L. (2004). Dietary restraint and heightened reactivity to food. Physiol Behav, 81(1), 85-90.

Buss, K. A., Schumacher, J. R., Dolski, I., Kalin, N. H., Goldsmith, H. H. \& Davidson, R. J. (2003). Right frontal brain activity, cortisol, and withdrawal behavior in 6-month-old infants. Behav Neurosci, 117(1), 11-20.

Coan, J. A. \& Allen, J. J. (2003). Frontal EEG asymmetry and the behavioral activation and inhibition systems. Psychophysiology, 40(1), 106-114.

Cools, J., Schotte, D. E. \& McNally, R. J. (1992). Emotional arousal and overeating in restrained eaters. J Abnorm Psychol, 101(2), 348351.

Dallman, M. F. (2007). Modulation of stress responses: how we cope with excess glucocorticoids. Exp Neurol, 206(2), 179-182.

Dallman, M. F., Akana, S. F., Strack, A. M., Hanson, E. S. \& Sebastian, R. J. (1995). The neural network that regulates energy balance is responsive to glucocorticoids and insulin and also regulates HPA axis responsivity at a site proximal to CRF neurons. Ann NY Acad Sci, 771, 730-742. 
Dallman, M. F., Akana, S. F., Strack, A. M., Scribner, K. S., Pecoraro, N., LA Fleur, S. E., ET AL. (2004). Chronic stress-induced effects of corticosterone on brain: direct and indirect. Ann NY Acad Sci, 1018, 141-150.

Dallman, M. F., Pecoraro, N., Akana, S. F., La Fleur, S. E., Gomez, F., Houshyar, H., ET AL. (2003). Chronic stress and obesity: a new view of "comfort food". Proc Natl Acad Sci USA, 100(20), 11696-11701.

Dallman, M. F., Pecoraro, N. C., La Fleur, S. E., Warne, J. P., Ginsberg, A. B., Akana, S. F., ET AL. (2006). Glucocorticoids, chronic stress, and obesity. Prog Brain Res, 153, 75-105.

Davidson, R. J. (1998). Anterior electrophysiological asymmetries, emotion, and depression: conceptual and methodological conundrums. Psychophysiology, 35(5), 607-614.

Davidson, R. J. (2002). Anxiety and affective style: role of prefrontal cortex and amygdala. Biol Psychiatry, 51(1), 68-80.

Davidson, R. J. (2004). What does the prefrontal cortex "do" in affect: perspectives on frontal EEG asymmetry research. Biol Psychol, 67(1-2), 219-233.

Davidson, R. J., Ekman, P., Saron, C. D., Senulis, J. A. \& Friesen, W. V. (1990). Approach-withdrawal and cerebral asymmetry: emotional expression and brain physiology. I. J Pers Soc Psychol, 58(2), 330-341.

Davidson, R. J., Lewis, D. A., Alloy, L. B., Amaral, D. G., Bush, G., Cohen, J. D., ET AL. (2002). Neural and behavioral substrates of mood and mood regulation. Biol Psychiatry, 52(6), 478-502.

Davidson, R. J., Marshall, J. R., Tomarken, A. J. \& Henriques, J. B. (2000). While a phobic waits: regional brain electrical and autonomic activity in social phobics during anticipation of public speaking. Biol Psychiatry, 47(2), 85-95.

Davidson, R. J., Pizzagalli, D., Nitschke, J. B. \& Putnam, K. (2002). Depression: perspectives from affective neuroscience. Annu Rev Psychol, 53, 545-574.

Davis, C., Curtis, C., Tweed, S. \& Patte, K. (2007). Psychological factors associated with ratings of portion size: relevance to the risk profile for obesity. Eat Behav, 8(2), 170-176.

Davis, C. \& Fox, J. (2008). Sensitivity to reward and body mass index (BMI): evidence for a non-linear relationship. Appetite, 50(1), 4349. 
Davis, C., Levitan, R. D., Kaplan, A. S., Carter, J., Reid, C., Curtis, C., ET AL. (2008). Reward sensitivity and the D2 dopamine receptor gene: A case-control study of binge eating disorder. Prog Neuropsychopharmacol Biol Psychiatry, 32(3), 620-628.

Davis, C., Patte, K., Levitan, R., Reid, C., Tweed, S. \& Curtis, C. (2007). From motivation to behaviour: a model of reward sensitivity, overeating, and food preferences in the risk profile for obesity. Appetite, 48(1), 12-19.

Davis, C., Strachan, S. \& Berkson, M. (2004). Sensitivity to reward: implications for overeating and overweight. Appetite, 42(2), 131138.

Epel, E., Lapidus, R., McEwen, B. \& Brownell, K. (2001). Stress may add bite to appetite in women: a laboratory study of stress-induced cortisol and eating behavior. Psychoneuroendocrinology, 26(1), 37-49.

Finlayson, G., King, N. \& Blundell, J. E. (2007). Liking vs. wanting food: importance for human appetite control and weight regulation. Neurosci Biobehav Rev, 31(7), 987-1002.

Gibson, E. L. (2006). Emotional influences on food choice: sensory, physiological and psychological pathways. Physiol Behav, 89(1), 53-61.

Gluck, M. E., Geliebter, A., Hung, J. \& Yahav, E. (2004). Cortisol, hunger, and desire to binge eat following a cold stress test in obese women with binge eating disorder. Psychosom Med, 66(6), 876881.

Grabenhorst, F., Rolls, E. T. \& Bilderbeck, A. (2007). How Cognition Modulates Affective Responses to Taste and Flavor: Top-down Influences on the Orbitofrontal and Pregenual Cingulate Cortices. Cereb Cortex.

Grabenhorst, F., Rolls, E. T., Margot, C., da Silva, M. A. \& Velazco, M. I. (2007). How pleasant and unpleasant stimuli combine in different brain regions: odor mixtures. J Neurosci, 27(49), 1353213540.

Greeno, C. G., Wing, R. R. \& Shiffman, S. (2000). Binge antecedents in obese women with and with out binge eating disorder. Journal of Consulting and Clinical Psychology, 68, 95-102.

Guerrieri, R., Nederkoorn, C. \& Jansen, A. (2008). The interaction between impulsivity and a varied food environment: its influence on food intake and overweight. Int J Obes (Lond), 32(4), 708-714.

Heatherton, T. F., Herman, C. P. \& Polivy, J. (1991). Effects of physical threat and ego threat on eating behavior. J Pers Soc Psychol, 60(1), 138-143. 
Heinrichs, S. C., Menzaghi, F., Merlo Pich, E., Britton, K. T. \& Коов, G. F. (1995). The role of CRF in behavioral aspects of stress. Ann NY Acad Sci, 771, 92-104.

Helmreich, D. L., Parfitt, D. B., Lu, X. Y., Akil, H. \& Watson, S. J. (2005). Relation between the hypothalamic-pituitary-thyroid (HPT) axis and the hypothalamic-pituitary-adrenal (HPA) axis during repeated stress. Neuroendocrinology, 81(3), 183-192.

Herman, C. P. \& Mack, D. (1975). Restrained and unrestrained eating. J Pers, 43(4), 647-660.

Herman, C. P. \& Polivy, J. (1975). Anxiety, restraint, and eating behavior. J Abnorm Psychol, 84(6), 66-72.

Herman, C. P. \& Polivy, J. (1980). Restrained Eating. In A. Stunkard (Ed.), Obesity (pp. 208-225). Philadelphia: Saunders.

Hughes, J. W., Watkins, L., Blumenthal, J. A., Kuhn, C. \& Sherwood, A. (2004). Depression and anxiety symptoms are related to increased 24-hour urinary norepinephrine excretion among healthy middle-aged women. J Psychosom Res, 57(4), 353-358.

Jacobi, C., Hayward, C., de Zwaan, M., Kraemer, H. C. \& Agras, W. S. (2004). Coming to terms with risk factors for eating disorders: application of risk terminology and suggestions for a general taxonomy. Psychol Bull, 130(1), 19-65.

Jansen, A., Merckelbach, H., Oosterlaan, J., Tuiten, A. \& van den Hout, M. (1988). Cognitions and self-talk during food intake of restrained and unrestrained eaters. Behav Res Ther, 26(5), 393-398.

Jeвb, S. A. (1997). Aetiology of obesity. Br Med Bull, 53(2), 264-285.

Kalin, N. H., Larson, C., Shelton, S. E. \& Davidson, R. J. (i998). Asymmetric frontal brain activity, cortisol, and behavior associated with fearful temperament in rhesus monkeys. Behav Neurosci, 112(2), 286-292.

Keel, P. K., Baxter, M. G., Heatherton, T. F. \& Joiner, T. E., Jr. (2007). A 20-year longitudinal study of body weight, dieting, and eating disorder symptoms. J Abnorm Psychol, 116(2), 422-432.

Koo-Loeb, J. H., Costello, N., Light, K. C. \& Girdler, S. S. (2000). Women with eating disorder tendencies display altered cardiovascular, neuroendocrine, and psychosocial profiles. Psychosom Med, 62(4), 539-548.

Kringelbach, M. L., O’Doherty, J., Rolls, E. T. \& Andrews, C. (2003). Activation of the human orbitofrontal cortex to a liquid food stimulus is correlated with its subjective pleasantness. Cereb Cortex, 13(10), 1064-1071. 
Krolak-Salmon, P., Fischer, C., Vighetto, A. \& Mauguiere, F. (2001). Processing of facial emotional expression: spatio-temporal data as assessed by scalp event-related potentials. Eur J Neurosci, 13(5), 987-994.

Krysiak, R., Obuchowicz, E. \& Herman, Z. S. (2000). Role of corticotropin-releasing factor (CRF) in anxiety. Pol J Pharmacol, 52(1), 15-25.

Marin, P., Darin, N., Amemiya, T., Andersson, B., Jern, S. \& Bjorntorp, P. (1992). Cortisol secretion in relation to body fat distribution in obese premenopausal women. Metabolism, 41(8), 882-886.

Nasser, J. (2001). Taste, food intake and obesity. Obes Rev, 2(4), 213-218.

Nederkoorn, C., Braet, C., Van Eijs, Y., Tanghe, A. \& Jansen, A. (2006). Why obese children cannot resist food: the role of impulsivity. Eat Behav, 7(4), 315-322.

Northoff, G. \& Bermpohl, F. (2004). Cortical midline structures and the self. Trends Cogn Sci, 8(3), 102-107.

Northoff, G., Heinzel, A., de Greck, M., Bermpohl, F., Dobrowolny, H. \& Panksepp, J. (2006). Self-referential processing in our brain-A meta-analysis of imaging studies on the self. Neuroimage, 31(1), 440-457.

Olff, M., Guzelcan, Y., de Vries, G. J., Assies, J. \& Gersons, B. P. (2006). HPA- and HPT-axis alterations in chronic posttraumatic stress disorder. Psychoneuroendocrinology, 31(10), 1220-1230.

Pardon, M. C., Ma, S. \& Morilak, D. A. (2003). Chronic cold stress sensitizes brain noradrenergic reactivity and noradrenergic facilitation of the HPA stress response in Wistar Kyoto rats. Brain Res, 971(1), 55-65.

Pecina, S. (2008). Opioid reward 'liking' and 'wanting' in the nucleus accumbens. Physiol Behav, 94(5), 675-680.

Pecina, S. \& Berridge, K. C. (2000). Opioid site in nucleus accumbens shell mediates eating and hedonic 'liking' for food: map based on microinjection Fos plumes. Brain Res, 863(1-2), 71-86.

Pecina, S., Schulkin, J. \& Berridge, K. C. (2006). Nucleus accumbens corticotropin-releasing factor increases cue-triggered motivation for sucrose reward: paradoxical positive incentive effects in stress? BMC Biol, 4, 8.

Pizzagalli, D. A., Sherwood, R. J., Henriques, J. B. \& Davidson, R. J. (2005). Frontal brain asymmetry and reward responsiveness: a source-localization study. Psychol Sci, 16(10), 805-813.

Polivy, J. \& Herman, C. P. (1999). Distress and eating: why do dieters overeat? Int J Eat Disord, 26(2), 153-164. 
Polivy, J., Herman, C. P. \& McFarlane, T. (1994). Effects of anxiety on eating: does palatability moderate distress-induced overeating in dieters? J Abnorm Psychol, 103(3), 505-510.

Rennie, K. L., Johnson, L. \& JebB, S. A. (2005). Behavioural determinants of obesity. Best Pract Res Clin Endocrinol Metab, 19(3), 343-358.

Rolls, E. T. (1996). The orbitofrontal cortex. Philos Trans $R$ Soc Lond B Biol Sci, 351(1346), 1433-1443; discussion 1433-1434.

Rolls, E. T. (2004). The functions of the orbitofrontal cortex. Brain Cogn, 55(1), 11-29.

Rosmond, R. (2003). Stress induced disturbances of the HPA axis: a pathway to Type 2 diabetes? Med Sci Monit, 9(2), RA, 35-39.

Schachter, S. (1968). Obesity and eating. Internal and external cues differentially affect the eating behavior of obese and normal subjects. Science, 161(843), 751-756.

Scholten, M. R., van Honk, J., Aleman, A. \& Kahn, R. S. (2006). Behavioral inhibition system (BIS), behavioral activation system (BAS) and schizophrenia: relationship with psychopathology and physiology. J Psychiatr Res, 40(7), 638-645.

Schotte, D. E., Cools, J. \& McNally, R. J. (1990). Film-induced negative affect triggers overeating in restrained eaters. J Abnorm Psychol, 99(3), 317-320.

Shekhar, A., Truitt, W., Rainnie, D. \& Sajdyk, T. (2005). Role of stress, corticotrophin releasing factor (CRF) and amygdala plasticity in chronic anxiety. Stress, 8(4), 209-219.

SiLva, J. R. (2002). Versión en español de la escala de restricción alimentaria (Restraint Scale). Unpublished manuscript.

SiLva, J. R. (2003). Biología de la regulación emocional: Su impacto en la psicología del afecto y la psicoterapia. Terapia Psicológica, 21, 163-172.

Silva, J. R. (2004). Diferenciación emocional y psicopatología: la hipótesis de acentuación somática. Revista Electrónica de Motivación y Emoción, 7(2), 1-16.

Silva, J. R. (2005a). Ansiedad y sobrealimentación. Investigación y Ciencia, 341, 33-34.

Silva, J. R. (2005b). Asimetrías frontales en dietantes crónicos, Informe de Investigación Diploma de Estudios Avanzados. Madrid: Universidad Complutense de Madrid.

SiLva, J. R. (2005c). Asimetrías funcionales frontales en el trastorno depresivo mayor. Revista Chilena de Neuropsiquiatría, 43, 305-313.

SiLva, J. R. (2005d). Regulación emocional y psicopatología: El modelo de vulnerabilidad/resiliencia. Revista Chilena de Neuropsiquiatría, 43, 201-209. 
Silva, J. R. (2007a). Estilo afectivo en dietantes crónicos: Una aproximación psicofisiológica. Universidad Complutense de Madrid, Madrid.

Silva, J. R.(2007b). Sobrealimentación inducida por la ansiedad, Parte I: Evidencia conductual, afectiva, metabólica y endocrina. Terapia Psicológica, 25, 112-134.

Silva, J. R. (2008a). Restricción alimenticia y sobrealimentación: Un modelo de la neurociencia afectiva. Revista Médica de Chile, 136, $1336-1342$.

Silva, J. R. (2008b). Sobrealimentación inducida por la ansiedad, Parte II: Un marco de referencia neurocientífico para el desarrollo de técnicas psicoterapéuticas. Terapia Psicológica 3(1), 99-115.

Silva, J. R. (en prensa). Consistencia interna y validez factorial de la versión en español de la escala revisada de restricción alimentaria. Revista Chilena de Nutrición.

Silva, J. R., Jiménez, M., Sapunar, J. y Larenas, G. (2007). Diferenciación cortical anormal de expresiones faciales emocionales en dietantes crónicos. Paper presentado en el XVIII Congreso Chileno de Endocrinología y Diabetes.

Silva, J. R., Livacic-Rojas, P. \& Slachevsky, A. (2006). [Differences among chronic restrained eaters: the influence of motivational systems]. Revista Médica de Chile, 134(6), 735-742.

Silva, J. R. \& Pizzagalli, D. (2006). Cortical response to food stimulus in restrained eaters under ego-threat anxiety: an ERP source localization study. Psychophysiology, 43, S92.

Silva, J. R., Pizzagalli, D. A., Larson, C. L., Jackson, D. C. \& Davidson, R. J. (2002). Frontal brain asymmetry in restrained eaters. J Abnorm Psychol, 111(4), 676-681.

Silva, J. R. \& Slachevsky, A. (2005). Modulación de la actividad de la corteza prefrontal como mecanismo putativo del cambio en psicoterapia. International Journal of Clinical and Health Psychology, 5, 609-625.

Smagin, G. N. \& Dunn, A. J. (2000). The role of CRF receptor subtypes in stress-induced behavioural responses. Eur J Pharmacol, 405(13), 199-206.

Small, D. M., Zatorre, R. J., Dagher, A., Evans, A. C. \& JonesGotman, M. (2001). Changes in brain activity related to eating chocolate: from pleasure to aversion. Brain, 124(Pt 9), 1720-1733.

Spencer, J. A. \& Fremouw, W. J. (1979). Binge eating as a function of restraint and weight classification. J Abnorm Psychol, 88(3), 262-267.

Starkman, M. N., Cameron, O. G., Nesse, R. M. \& Zelnik, T. (1990). Peripheral catecholamine levels and the symptoms of anxiety: studies in patients with and without pheochromocytoma. Psychosom Med, 52(2), 129-142. 
Sutton, S. K. \& Davidson, R. J. (2000). Prefrontal brain electrical asymmetry predicts the evaluation of affective stimuli. Neuropsychologia, 38(13), 1723-1733.

TAkahashi, L. K. (2002). CRF(2) Receptors: An Emerging Role in Anxiety. Drug News Perspect, 15(2), 97-101.

Todorovic, C., Jahn, O., Tezval, H., Hippel, C. \& Spiess, J. (2005). The role of CRF receptors in anxiety and depression: implications of the novel CRF1 agonist cortagine. Neurosci Biobehav Rev, 29(8), 1323-1333.

Tomarken, A. J. \& Keener, A. M. (1998). Frontal brain asymmetry and depression: A self-regulatory perspective. Cognition \& Emotion, $12,387-420$.

Torres, C. W., Silva, J. R. \& Silva, F. L. (2007). [Evaluation of personal traits, self, schizophrenia and cortical mid line structures]. Revista Médica de Chile, 135(5), 653-660.

Tuschen-Caffier, B. \& Vogele, C. (1999). Psychological and physiological reactivity to stress: an experimental study on bulimic patients, restrained eaters and controls. Psychother Psychosom, 68(6), 333-340.

Van Strien, T. \& Ouwens, M. A. (2007). Effects of distress, alexithymia and impulsivity on eating. Eat Behav, 8(2), 251-257.

Wang, G. J., Volkow, N. D., Thanos, P. K. \& Fowler, J. S. (2004). Similarity between obesity and drug addiction as assessed by neurofunctional imaging: a concept review. J Addict Dis, 23(3), 39-53.

Ward, A. \& MAnn, T. (2000). Don't mind if I do: disinhibited eating under cognitive load. J Pers Soc Psychol, 78(4), 753-763.

Wardle, J., Steptoe, A., Oliver, G. \& Lipsey, Z. (2000). Stress, dietary restraint and food intake. J Psychosom Res, 48(2), 195-202.

Waters, A., Hill, A. \& WAller, G. (2001). Bulimics' responses to food cravings: is binge-eating a product of hunger or emotional state? Behaviour Research And Therapy, 39(8), 877-886.

Wyatt, R. J., Portnoy, B., Kupfer, D. J., Snyder, F. \& Engelman, K. (1971). Resting plasma catecholamine concentrations in patients with depression and anxiety. Arch Gen Psychiatry, 24(1), 65-70.

Fecha de Recepción de artículo: 19 de agosto de 2009.

Fecha de Aceptación de artículo: 17 de noviembre de 2009. 
